\title{
Germinación de semillas de Ficus insipida (Moraceae) defecadas por tucanes (Ramphastos sulfuratus) y monos araña (Ateles geoffroyi)
}

\author{
Laura E. Domínguez-Domínguez ${ }^{1}$, Jorge E. Morales-Mávil ${ }^{1}$ \& Juan Alba-Landa ${ }^{2}$ \\ 1 Instituto de Neuroetología, Universidad Veracruzana, Apdo. Postal 566, Xalapa, Veracruz, México 91000. Tel: (228) \\ 8125748, Fax: (228) 8135157; ledominguez@uv.mx, jormorales@uv.mx \\ 2 Instituto de Genética Forestal, Universidad Veracruzana, Apdo. Postal 551, Xalapa, Veracruz, México 91000. Tel/Fax: \\ (228) 121357; genfor@uv.mx
}

Recibido 20-V-2003. Corregido 28-IX-2004. Aceptado 08-VI-2005.

\begin{abstract}
Germination of Ficus insipida (Moraceae) seeds from toucan (Ramphastos sulfuratus) and spider monkey (Ateles geoffroyi) feces. To test the null hypothesis that two vertebrate fructivores, toucans (Ramphastos sulfuratus) and spider monkeys (Ateles geoffroyi), are equally specialized in germinating Ficus insipida seeds after these have passed through their digestive tracts, we fed fruits to captive individuals. We extracted seeds from feces and placed them on filter paper in petri dishes under controled light, temperature and humidity. Control seeds had not passed through a digestive tract. We found that a greater proportion of seeds from $A$. geofroyi $(65 \%)$ ) germinated (R. sulfuratus: $4 \%$ ). The germinative value was also greater in seeds from monkey feces (rate $=13.76$; toucan 0.046 ; control group 0.172 ). If, despite individual variability of seeds and dispersers, future studies continue to show that $A$. geoffroyi favors germination more (maybe because of a longer digestion time), this would indicate that diet specialization is not necessarily related to dispersal efficiency. Rev. Biol. Trop. 54(2): 387-394. Epub 2006 Jun 01.
\end{abstract}

Key words: Ramphastos sulfuratus, Ateles geoffroyi, Ficus, germination, dispersal, frugivory.

La mayoría de los árboles tropicales requieren de animales para dispersar sus semillas (Howe 1980). Existen muchos factores que actúan en la determinación de los patrones de dispersión de las plantas; los frugívoros son un elemento importante en este proceso. Las interacciones entre los animales que se alimentan de frutos y las plantas son de particular relevancia para entender la dinámica de la selva; por tanto, los frugívoros tropicales juegan un papel primordial en la sucesión secundaria y en el mantenimiento de la heterogeneidad de los bosques tropicales. En algunas ocasiones, el paso por el tracto digestivo del animal contribuye a disminuir el tiempo para que la semilla se active; sin embargo, en otros casos, puede afectar su viabilidad; es probable que esta acción sea ocasionada por los jugos gástricos que contenga el tracto digestivo del individuo, ya que en algunas semillas, éste acelera la escarificación, pero en otras, puede afectar negativamente al embrión (Howe y Estabrook 1977).

Dentro de la gran variedad de especies de árboles existentes en los bosques tropicales, las del género Ficus son de las más importantes. Las especies pertenecientes a este género son utilizadas como recurso alimentario por muchos frugívoros; por ejemplo, dentro de los mamíferos, se encuentran los monos aulladores, monos araña (Milton 1980, Glander 1981, Estrada 1984, Estrada y Coates-Estrada 1984, 1986a, 1986b, Serio-Silva 1995, DomínguezDomínguez 1994, Julliot 1996, Rodríguez-Luna et al. 1995, 2003, Dew y Wright 1998, Bravo y Zunino 2000, García-Orduña 2003), algunas 
especies de murciélagos (Artibeus sp., Sturnira sp., Glossophaga sp.) (Howe y Primack 1975, Orozco-Segovia et al. 1985, Coates-Estrada y Estrada 1986, Galindo-González et al. 2000), los tlacuaches (Marmosa mexiana, Didelphis marsupialis, Philander opossum), algunas ardillas (Sciurus aureogaster, Sciurus deppei), el puerco espín (Sphiguirus mexicanus), los seretes (Dasyprocta mexicana), el cacomixtle (Bassariscus sumichrasti) y las martuchas (Potos flavus), entre otros (Coates-Estrada y Estrada 1986). Asimismo, varias especies de aves, como los tucanes ( $R$. sulfuratus) y los trogones (Trogon spp.) (van Dorp 1985, Remsen et al. 1993, Pérez 1996) y algunos reptiles (Morales-Mávil 1997), consumen este recurso.

Muchos de los frugívoros consumidores de Ficus pueden, asimismo, actuar como agentes dispersores o como depredadores de las semillas. Para evaluar el potencial de un animal como dispersor de semillas, se deberá considerar al menos dos componentes: la cantidad y la calidad de dispersión (Schupp 1993). En este trabajo comparamos el efecto de algunos componentes que muestran la calidad de la dispersión de semillas: porcentaje de germinación, velocidad de germinación y la capacidad germinativa de semillas de $F$. insipida consumidas y defecadas por monos araña (A. geoffroyi) y por tucanes ( $R$. sulfutarus).

Ateles y Ramphastos han sido considerados frugívoros especialistas (Howe 1993, Peres 1994, Stevenson et al. 2002). Se conoce que la dieta de Ateles está basada principalmente en frutos carnosos y es complementada con una variable proporción de hojas y flores (Estrada y Coates-Estrada 1986b, Van Roosmal y Klein 1988, Peres 1994, García-Orduña 2003); mientras que la de $R$. sulfuratus consiste básicamente en frutos y ocasionalmente ingiere materia animal (van Dorp 1985, Remsen et al. 1993). Por lo cual, esperaríamos una efectividad similar en la capacidad germinativa de las semillas defecadas por ambos vertebrados. Esta hipótesis la formulamos considerando las ideas prevalecientes de que existe una fuerte relación entre las plantas y sus dispersores potenciales, de tal manera que entre más especialista sea el frugívoro, mayor será su potencial como dispersor (Herrera 1986, Jordano 1995).

El presente estudio pretende analizar la contribución que tienen para la germinación de semillas de F. insipida, dos especies de frugívoros: el tucán ( $R$. sulfuratus) y el mono araña ( $A$. geoffroyi). Cada uno de estos vertebrados, por provenir de grupos distintos (aves y mamíferos), tienen hábitos y características digestivas diferentes que permiten la comparación sobre su potencial como agentes dispersores de semillas.

\section{MATERIALES Y MÉTODOS}

Se mantuvieron en cautiverio seis individuos adultos de dos especies de vertebrados frugívoros: tres tucanes ( $R$. sulfuratus) y tres monos araña ( $A$. geoffroyi), en las instalaciones del "Parque de Flora y Fauna Silvestre Tropical" (PAFFASIT), reserva ecológica de la Universidad Veracruzana, ubicada en la región de Los Tuxtlas, Veracruz, México; los animales estuvieron confinados en jaulas individuales de $2 \times 1.5 \times 1.5 \mathrm{~m}$ para los tucanes y jaulas de $3 \times$ $4 \times 2 \mathrm{~m}$ para los monos. Estos animales provienen de decomisos realizados por la PROFEPA $\mathrm{y}$ han sido mantenidos en cautiverio por espacio de 3 a 5 años. Los animales han sido alimentados diariamente con una combinación de frutos silvestres y cultivados.

El ensayo consistió en ofrecer siconos maduros (en los sucesivo frutos) de $F$. insipida a cada uno de los animales. Los frutos fueron colectados aleatoriamente de por lo menos dos individuos arbóreos ubicados en sitios periféricos al PAFFASIT y proporcionados a los animales el mismo día de su colecta. Los frutos fueron ofrecidos en recipientes de plástico, los mismos donde regularmente eran alimentados los animales. El número de frutos suministrados varió de 10 a 20 para los monos y de 15 a 20 para los tucanes, dependiendo de la aceptación de los animales. El ofrecimiento de los frutos se efectuó a las 09:00 horas durante dos días consecutivos.

Las heces fecales fueron recogidas inmediatamente después de ser excretadas. De tal 
manera que de los tucanes fueron colectadas no más de 35 minutos después de ser alimentados, mientras que las de monos se recogieron al menos 20 horas después del consumo de los frutos. Las heces fueron llevadas a laboratorio y secadas por algunos minutos a temperatura ambiente; inmediatamente después fueron separadas las semillas utilizando un microscopio estereoscópico, pinzas y agujas de disección.

Una vez separadas las semillas, se pusieron a germinar en cajas petri, utilizando sólo papel filtro como sustrato. El número de semillas depositadas en las cajas fue de $25 \mathrm{y}$ fueron utilizadas tres cajas por especie de vertebrado, cuantificando un total de 75 semillas provenientes de heces de $R$. sulfuratus y 75 de heces de $A$. geoffroyi. Las cajas fueron colocadas en repisas, cubiertas con un papel negro y mantenidas bajo un régimen de 12 horas luz, con un intervalo de temperatura de $19.2^{\circ} \mathrm{C}-26^{\circ} \mathrm{C}$ $\left(\mathrm{X}=22.1^{\circ} \mathrm{C}\right)$. Se procuró mantener siempre húmedo el sustrato de cada caja, mediante el riego diario o cada vez que se requería. Como grupo control se utilizaron semillas extraídas de frutos maduros cortadas directamente de los árboles, sin que hubieran pasado por el tracto digestivo de ningún animal. Las condiciones de germinación fueron las mismas que las usadas con las semillas provenientes de las excretas.

Para el análisis inicial de los datos se realizaron exámenes exploratorios de las variables de interés (latencia, porcentaje de germinación, velocidad de germinación y valor germinativo) por especie animal. Posteriormente, se realizó una prueba ANOVA de Rangos (KruskalWallis) para la comparación de la velocidad de germinación (utilizando como prueba post-hoc el Método de Dunn). La Chi-cuadrada se utilizó para detectar diferencias en la capacidad geminativa entre especies animales. El nivel de confianza para ambas pruebas fue del $95 \%$.

\section{RESULTADOS}

Los resultados muestran latencias similares para los tres grupos experimentales (17 días para la primera semilla separada de las heces de monos; 18 para las de tucán y 20 días para la proveniente del grupo testigo). Sin embargo, al finalizar el experimento la velocidad de germinación mostró diferencias significativas entre los tres grupos (ANOVA, $\mathrm{p} \leq 0.05$ ) (19.9 \pm 2.2 días para el mono araña, $21 \pm 5.3$ para el tucán y $24 \pm 3.4$ días para el grupo testigo) (Tabla 1). La prueba post-hoc, detectó significancia estadística entre los datos de los monos araña y los provenientes del grupo testigo (Dunn, $\mathrm{p} \leq 0.05$ ).

\section{CUADRO 1}

Estadísticas descriptivas de los ensayos de germinación realizados con semillas de $\mathrm{F}$. insipida, obtenidas de excretas de tucán, de mono araña y de testigo

$\begin{array}{cccc}\text { Parámetro } & \text { Heces } & \text { Heces } & \text { Grupo } \\ \text { de tucán } & \text { de mono } & \text { testigo }\end{array}$

$\begin{array}{lccc}\text { Latencia (días) } & 18 & 17 & 20 \\ \begin{array}{l}\text { Capacidad germinativa } \\ \text { (\% final de germinación) }\end{array} & 4 & 65.3 & 8.9 \\ \begin{array}{l}\text { Velocidad de } \\ \text { germinación } \\ \text { (días: } X \pm \text { DS) }\end{array} & 21.7 \pm 5.5 & 19.9 \pm 2.2 & 24.1 \pm 3.4 \\ \begin{array}{l}\text { Periodo de germinación } \\ \text { (días) }\end{array} & 10 & 10 & 8 \\ \text { Valor germinativo } & 0.046 & 13.757 & 0.172\end{array}$

La capacidad germinativa fue mayor en las semillas separadas de heces de mono araña: más del $65 \%$ vs. $4 \%$ del tucán y $8.3 \%$ del grupo testigo. Al realizarse las pruebas de significancia, se obtuvo que el porcentaje de germinación de las semillas extraídas de excretas de monos fue significativamente mayor que el registrado para las provenientes de excretas de tucanes $\left(\chi^{2}=59.6, \mathrm{p} \leq 0.05\right)$ y para las del grupo testigo $\left(\chi^{2}=55.2, p \leq 0.05\right)$. No se encontraron diferencias significativas entre los porcentajes de germinación de las semillas provenientes de las heces de tucán y las muestras testigo. Por consiguiente, el valor germinativo fue superior para las semillas provenientes de excretas de mono, registrando un índice de 13.76 (Tabla 1). 
Se calculó también el porcentaje acumulado de germinación (Fig. 1). Los resultados mostraron que las semillas consumidas por los monos araña empezaron a germinar antes que los otros dos grupos y de una forma relativamente constante durante 10 días, alcanzando el máximo porcentaje antes que los demás. Por su parte, las semillas separadas de las heces de tucán iniciaron su germinación un día después que las de los monos; sin embargo, después del cuarto día, se presentó un periodo en que ninguna semilla germinó, reanudándose la germinación hasta el día 11. Fueron las semillas del grupo testigo las que tardaron más tiempo en emerger (tres días después que el resto), presentando también un lapso de cinco días consecutivos en que no se observó la germinación (Fig. 1).

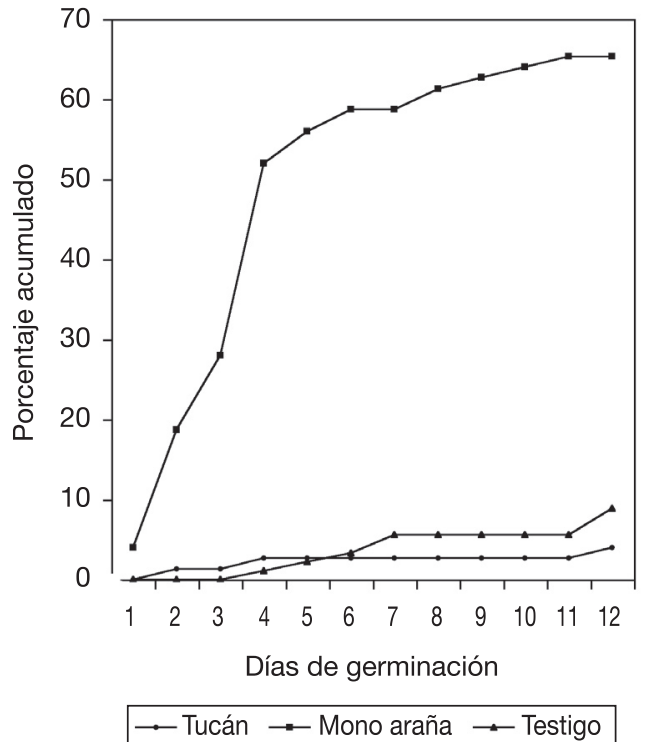

Fig. 1. Porcentaje acumulado de la germinación de semillas de F. insipida consumidas por tucanes, monos araña y provenientes de un grupo testigo.

\section{DISCUSIÓN}

Las variables de la calidad de germinación medidas en este trabajo, muestran el efecto neutral del tucán y el positivo del mono araña sobre la germinación de las semillas de $F$. insipida. Esto revela una diferencia en el efecto que pueden tener dos frugívoros especialistas sobre la germinación de una especie vegetal. Asimismo, es notable la marcada discrepancia entre la información existente acerca del potencial de los vertebrados frugívoros como agentes dispersores de semillas. En este estudio, el mono araña (A. geoffroyi) fue el vertebrado que benefició, en mayor medida, a la germinación de las semillas de $F$. insipida, sin embargo, existen trabajos que indican que esta especie no siempre favorece la germinación de semillas de Ficus Por ejemplo, Righini (2003) observó, en condiciones de laboratorio, que el porcentaje de germinación de semillas de Ficus perforata consumidas por $A$. geoffroyi fue sólo del $10.8 \%$, mucho menor al que registró para su grupo testigo $(71.3 \%)$; observando un tiempo inicial de germinación de 14 días. En otra especie de Ateles (A. belzebuth), Stevenson et al. (2002) no encontraron diferencias significativas en las latencias de germinación de las semillas de Ficus pertusa separadas de las heces de mono y las testigo, aunque sí en los porcentajes, siendo favorecidas las semillas consumidas por Ateles.

Si las semillas de una misma especie vegetal responden de diferente forma a tratamientos fisiológicos similares durante la endozoocoria (Stevenson et al. 2002), no es extraño entonces, que diferentes especies de Ficus desplieguen comportamientos germinativos desiguales al pasar por el tracto digestivo de Ateles. Si a esto anexamos el estado fisiológico de los animales, su dieta y las condiciones ambientales donde se lleva a cabo el proceso de germinación, las diferencias pueden ser explicadas.Las semillas de $F$. insipida pasaron poco tiempo por el tracto digestivo de los tucanes, en promedio 25 minutos; periodo similar a lo reportado en la literatura (33 minutos, Pérez 1996). Aparentemente, esta rápida evacuación de las semillas no fue suficiente para que el proceso digestivo tuviera un efecto favorable sobre su germinación, de tal manera que no se presentaron diferencias significativas en los parámetros germinativos evaluados entre las semillas separadas de las 
heces de tucán y las de la muestra testigo. Es probable que el reblandecimiento de la cubierta de la semilla no haya sido el óptimo para ayudar a mejorar la velocidad de su germinación y que el tratamiento que recibieron en el tracto digestivo de esta ave fue muy débil. En este sentido, se ha registrado en otras aves de tamaño similar, tiempos de digestión de varias horas, como es el caso del Ramphastos toco, un tucán considerado excelente dispersor de semillas de Ormosia arborea (Galletti 2002). No obstante, deberemos considerar lo mencionado por Levey (1986), quien dice que el tiempo que tarda una semilla en el tracto digestivo va a depender del tamaño de ésta, dado que las aves frugívoras presentan un mecanismo para separar la pulpa de la semilla. Dicho proceso es más rápido y fácil con semillas grandes que con pequeñas. Los frutos de Ficus, no sólo presentan semillas pequeñas (de 1 a $1.2 \mathrm{~mm}$ de largo y 0.8 a $1.1 \mathrm{~mm}$ de ancho) sino numerosas (entre 100 y 150), por lo cual los tucanes tardan más tiempo en la digestibilidad de sus semillas. Pérez (1996), encontró una relación inversa entre el tamaño de las semillas y el tiempo de digestión en $R$. sulphuratus.

Los resultados de la germinación de las semillas provenientes de las heces de tucán, difieren poco de lo reportado por Pérez (1996) con la misma especie de Ficus. Esta autora mencionó que el tiempo de germinación de semillas obtenidas de excretas de tucanes fue de 30 días en condiciones naturales y de 20 días en invernadero, este último similar a lo reportado en este trabajo (21.7 días). No obstante, sí se apreciaron diferencias en los porcentajes de germinación: $35 \%$ en condiciones naturales y $25 \%$ en invernadero para el trabajo de Pérez (1996) y sólo el 4\% para este estudio. Estos datos siguen mostrando la gran variación que puede existir en la germinación de semillas de una misma especie arbórea y que en las comparaciones que puedan establecerse, siempre se deberá considerar ese grado de error que implica la variación individual de los árboles y las diferentes temporadas de producción de frutos de éstos, además de los factores ya mencionados. Estos resultados muestran un efecto neutral del tucán sobre la germinación de semillas de F. insipida, lo cual sólo corresponde a una parte de lo que se considera la calidad de la dispersión (Schupp 1993), desconociendo todavía otras partes importantes, como son: la distancia y el sitio de deposición de excretas. Tomando en cuenta esto, algunos autores han considerado a $R$. sulfuratus como un importante dispersor de semillas de los bosques tropicales (van Dorp 1985, Pérez 1996).

Mckey (1975) menciona que si las semillas permanecen mucho tiempo en el tracto digestivo, existe el riesgo de que puedan ser excesivamente dañadas. Por supuesto que esto va a depender del tamaño de las semillas y la dureza de su testa, por lo cual, mientras que algunas pueden verse afectadas negativamente por un tiempo excesivo en el tracto, otras pueden encontrar un punto óptimo para germinar. Es probable que para las semillas de $F$. insipida tratadas en este estudio, las horas que tardaron en el tracto digestivo del mono araña antes de ser evacuadas, fue más favorable que los pocos minutos que tardó la digestión del tucán, donde al parecer no fue suficiente para favorecer el adelgazamiento de la testa. El mismo McKey (1975) asegura que si las semillas consumidas son evacuadas de forma rápida (como en el caso del tucán), existe el peligro de que sean depositadas cerca o abajo del árbol progenitor, reduciéndose así la dispersión. Las semillas de Ficus y de otras especies cuyos frutos son parecidos en tamaño (como Cecropia obtusifolia), pueden ser dispersadas a mayores distancias del árbol progenitor, por permanecer más tiempo en el tracto digestivo del frugívoro dispersor.

Si bien, tanto el mono araña como el tucán han sido considerados frugívoros especialistas, nuestros resultados no apoyan la hipótesis de que ambas especies tengan efectos similares sobre la calidad de dispersión de las semillas de F. insipida. No obstante, ambos frugívoros son habitantes de los bosques tropicales y su presencia y alta movilidad, tanto en vegetación continua como en hábitat fragmentado (van Dorp 1985, Estrada y Coates-Estrada 1986b, Van Roosmal y Klein 1988, García-Orduña 
2003), les da la posibilidad de depositar las semillas que consumen lejos de los árboles progenitores, ayudando con ello a la dispersión de las especies vegetales.

\section{AGRADECIMIENTOS}

Al Consejo Nacional de Ciencia y Tecnología (CONACYT, Registro No. 175069) por la beca otorgada al primer autor. A Marlon Gil por su ayuda en la revisión del Abstract y a Ernesto Rodríguez Luna por las facilidades otorgadas para la realización del trabajo.

\section{RESUMEN}

Con el objetivo de evaluar y comparar el efecto que tiene el paso de las semillas del higo Ficus insipida Willd (Moraceae), por el tracto digestivo de dos especies de vertebrados frugívoros, se ofrecieron frutos a individuos cautivos de tucán (Ramphastos sulfuratus: Ramphastidae) y de mono araña (Ateles geoffroyi: Cebidae), en la hipótesis de que siendo ambos frugívoros especialistas deberían favorecer de manera similar a la germinación de semillas de esta especie arbórea. De las heces se separaron las semillas y se pusieron a germinar en cajas petri en condiciones controladas de luz, temperatura y humedad, con una muestra de semillas testigo que no pasaron por el tracto digestivo de ningún animal. Se calculó la latencia, la capacidad y velocidad de geminación, y el valor germinativo de las semillas de cada grupo experimental. El $65 \%$ de las semillas de frutos de $F$. insipida consumidos por $A$. geofroyi germinaron con éxito, mientras que de las obtenidas de las heces de tucán, apenas el 4\% logró germinar. El valor germinativo fue mayor en las semillas extraídas de excretas de mono (índice=13.76), en comparación con las de tucán (0.046) y las del grupo testigo (0.172). A. geoffroyi fue la especie que favoreció más la germinación de semillas de $F$. insipi$d a$, siendo probable que esta condición se relacione con el mayor tiempo de digestión de este primate. Deberá considerarse también la dificultad de establecer siempre concordancia con estos resultados, debido a la gran variabilidad en la capacidad germinativa de las semillas de individuos arbóreos, a la diferencia entre las cosechas y periodos de fructificación, así como en la madurez y estado fisiológico de los animales utilizados en las pruebas. Se concluye que la especialización alimentaria no necesariamente se relaciona con la efectividad de la calidad de la dispersión.

Palabras clave: Ramphastos sulfuratus, Ateles geoffroyi, Ficus, germinación, dispersión, frugivoría.

\section{REFERENCIAS}

Bravo, S.P. \& G.E. Zunino. 2000. Germination of sedes from three species dispersed by black howler monkyes (Alouatta caraya). Folia Primatol. 71: 342-345.

Coates-Estrada, R. \& A. Estrada. 1986. Manual de identificación de campo de los mamíferos de la Estación de Biología Tropical "Los Tuxtlas". Instituto de Biología, UNAM. México, D.F. 151 p.

Dew, J.L. \& P. Wrigth. 1998. Frugivory and seed dispersal by four species of primates in Madagascar's eastern rain forest. Biotropica 30(3): 425-437.

Domínguez-Domínguez, L.E. 1994. Preferencias alimenticias y comportamiento agonístico de Alouatta palliata en condiciones de cautiverio. La Ciencia y El Hombre. 18: 105-125.

Estrada, A. 1984. Resource use by howler monkeys (Alouatta palliata) in the rain forest of Los Tuxtlas, Veracruz, Mexico. Int. J. Primatol. 5: 105-131.

Estrada, A. \& R. Coates-Estrada. 1984. Fruti eating and seed dispersal by howling monkeys (Alouatta palliata) in a tropical rain forest of Los Tuxtlas, Mexico. Amer. J. Primatol. 6: 77-91.

Estrada, A. \& R. Coates-Estrada. 1986a. Fruting and frugivores at a strangler fig in the tropical rain forest of Los Tuxtlas. J. Trop. Ecol. 2: 349-357.

Estrada, A. \& R. Coates-Estrada. 1986b. Frugivory by howling monkeys (Alouatta palliata) at Los Tuxtlas, Mexico: dispersal and fate of seeds. p. 93-140. In A. Estrada \& T.H. Fleming (eds.). Frugivores and seed dispersal. Dr. Junk, Dordtrech, Holanda.

Galetti, M. 2002. Seed dispersal of mimetic fruits: parasitism, mutualism, aposematism or exaptation? p. 177191. In D.J. Levey, W.R. Silva \& M. Galetti (eds.). Seed dispersal and frugivory: ecology, evolution and conservation. CABI, Nueva York, EEUU.

Galindo-González, J., S. Guevara \& V.J. Sosa. 2000. Batand Bird-generated seed rains at isolated trees in pastures in a tropical rainforest. Conserv. Biol. 14: 1693-1703.

García-Orduña, F. 2003. Comparación de las estrategias de forrajeo de Ateles geoffroyi vellerosus y Alouatta palliata mexicana, en un fragmento de selva en la Sierra de Santa Marta, Veracruz. Tesis de Maestría. Instituto de Neuroetología, Universidad Veracruzana. Xalapa, Veracruz, México. 59 p.

Glander, K. 1981. Feeding patterns in mantled howler monkeys. p. 231-257. In A.C. Kamil \& T.D. Sargent 
(eds.). Foraging behavior: ecological, ethological and psychological approaches. Gartland STPM, Nueva York, EEUU.

Herrera, C.M. 1986. Vertebrate-dispersed plants: why the don't behave the way they should. p. 5-18. In A. Estrada \& T.H. Fleming (eds.). Frugivores and seed dispersal. Dr. Junk, Dordtrech, Holanda.

Howe, H.F. 1980. Monkeys dispersal and waste of a neotropical fruit. Ecology 61: 944-959.

Howe, H.F. 1993. Specialized and generalized dispersal systems: where does "the paradigm" stand? Vegetation 107/108: 3-13.

Howe, H.F. \& R. Primack, 1975. Differential seed dispersal by birds of the tree Caesaria nitida (Flacourtiaceae). Biotropica 7: 278-283.

Howe, H.F. \& G.F. Estabrook. 1977. On intraespecific competition for avian dispersers in tropical trees. Amer. Natur. 111: 817-832.

Jordano, P. 1995. Angiosperm fleshy fruits and seed dispersers: a comparative analysis of adaptation and constraints in plant-animal interactions. Amer. Natur. 145: 163-191.

Julliot, C. 1996. Seed dispersal by red howling monkeys (Alouatta seniculus) in the tropical rain forest of French Guiana. Ecolog. Dispersal. p. 239-258.

Levey, D.J. 1986. Methods of seed processing by birds and seed deposition patterns. p. 147-158. In A. Estrada \& T.H. Fleming (eds.). Frugivores and seed dispersal.W. Junk. Dordrecht, Holanda.

McKey, D. 1975. The ecology of coevolved seed dispersal system. p. 159-191. In L.E. Gilbert \& P.H. Raven (eds.). Coevolution of animals and plants. University of Texas, Texas, EEUU.

Milton, K. 1980. The foraging strategy of howler monkeys (a study in primate economics). Columbia University, Nueva York, EEUU. 165 p.

Morales-Mávil, J.E. 1997. Viabilidad de semillas ingeridas por la iguana verde (Iguana iguana L.), en la zona de La Palma, región de Los Tuxtlas, Veracruz, México. Tesis de Maestría. Instituto de Neuroetología, Universidad Veracruzana. Xalapa, Veracruz, México. 66 p.

Orozco-Segovia, A., C., Vazquez-Yanes \& N. Correa. 1985. Interacciones entre una población de murciélagos de la especie Artibeus jamaicensis y la vegetación del área circundante, en la región de Los Tuxtlas, Veracruz. p. 365-377. In A. Gómez-Pompa \& S. Del
Amo (eds.). Investigación sobre la regeneración de selvas altas en Veracruz, México. Vol. II. INIREB y Alambra Mexicana, México, D.F.

Peres, C.A. 1994. Diet and feeding ecology of gray woolly monkeys (Lagothrix lagotricha cana) in central Amazonia: comparisons with other atelines. Int. J. Primatol. 15: 333-372.

Pérez V., B.L. 1996. Aspectos sobre la alimentación del tucan Ramphastos sulfuratus y su efecto sobre la germinación, dentro de la dispersión de semillas. Tesis de Licenciatura. Escuela Nacional de Estudios Profesionales, Campus Iztacala, UNAM. México, D.F. 112 p.

Remsen, J.V., M.A. Hyde \& A. Chapman. 1993. The diets of netropical trogons, momots, barbets and toucans. Condor. 95: 178-192.

Righini, N. 2003. Germinación de semillas de Ficus perforata ingeridas por monos aulladores (Alouatta palliata mexicana) y monos araña (Ateles geoffroyi vellerosus). Tesis de Maestría. Instituto de Ecología, A.C. Xalapa, Veracruz, México. 46 p.

Rodríguez-Luna, E., L.E. Domínguez-Domínguez, J.E. Morales-Mávil \& M. Martínez-Morales. 2003. Foraging strategy changes in an Alouatta palliata mexicana troop released on an island. p. 229-247. In L.K. Marsh (ed.). Primates in fragments: ecolgy and conservation. Kluwer Academic / Plenum, Nueva York, EEUU.

Rodríguez-Luna, E., M. Martínez-Morales, J.C. SerioSilva \& L.E. Domínguez-Domínguez. 1995. Forrajeo del mono aullador (Alouatta palliata) en semilibertad. p. 133-148. In E. Rodríguez-Luna, L. Cortés-Ortiz \& J. Martínez-Contreras (eds.). Estudios primatológicos en México. Vol. II. Biblioteca de la Universidad Veracruzana. Xalapa, Veracruz, México.

Serio-Silva, J.C. 1995. Patrón diario de actividades y hábitos alimenticios de Alouatta palliata en semilibertad. p. 149-172. In E. Rodríguez-Luna, L. Cortés-Ortiz \& J. Martínez-Contreras (eds.). Estudios primatológicos en México. Vol. II. Biblioteca de la Universidad Veracruzana. Xalapa, Veracruz, México.

Schupp, E.W. 1993. Quantity, quality and the effectiveness of seed dispersal by animals. Vegetation 107/108: 15-29.

Stevenson, P.R., M.C. Castellanos, J.C. Pizarro \& M. Garavito. 2002. Effect of seed dispersal by three ateline monkey species on seed germination at Tinigua National Park, Colombia. Int. J. Primatol. 23: 1187-1204. 
van Dorp, D. 1985. Frugivoría y dispersión de semillas por aves. p. 333-363. In A. Gómez-Pompa \& S. del Amo R. (eds.). Investigaciones sobre la regeneración de selvas altas en Veracruz, México. Vol. II. INIREB/ Alambra Mexicana, México, D.F. van Roosmalen, M.G. \& L.L. Klein. 1988. The spider monkeys genus Ateles. p. 455-537. In R.A. Mittermeir (ed.). Ecology and behavior of neotropical primates. Vol. 2. World Wildlife Fund. Washington, D.C., EEUU. 